\title{
Thermomechanical analysis of the surface vascular system - Application to the diabetic foot
}

\author{
by V. Serantoni ${ }^{*}$, F. Jourdan*, H. Louche*, A. Sultan** \\ * LMGC, Univ. Montpellier, CNRS, Rue St-Priest, 34095, Montpellier, France, vincent.serantoni@gmail.com \\ montpellier.fr \\ ** CHU Lapeyronie, Nutrition-Diabète, Av. Doyen G. Giraud, 34090, Montpellier, France, a-sultan@chu-
}

\begin{abstract}
Diabetes Mellitus (DM) is a huge public health-care chronic disease issue, with about 415 millions of patients worldwide in 2015. DM is a serious pathology, especially through its complications and their severity, as one, called diabetic foot, which can lead to a lower limb amputation. The aim of the present study is to propose a dynamic method to classify the foot state through a thermal method. This one uses infrared imaging recorded after the application of a local cold stimulus and a bio-heat model. The model's thermophysical and micro-perfusion parameters are identified and compared to the patients' clinical classification.
\end{abstract}

\section{Introduction}

The worst complication of the Diabetic Foot (DF) is the lower extremity ulcerations and infection. This complication is especially leading to the loss of sensitivity in the foot. The relationship between DM and lower extremity amputation is well recognized. This is a complex disease with a medical, social (lifestyle), human (quality of life) and economic (high cost for the health care systems) main issue. DF can lead to a long full hospitalisation and even to a traumatic lower limb amputation. The current technique for classifying the DF are qualitative, so it can be very difficult for the practitioner to adapt and anticipate the evolution of the foot of a patient. The use of an infrared (IR) camera and sensors in the medical area exists since many years [1]. The non-invasive and non-ionising specificity of this technology, for the patient and the practitioner, is very interesting.

The main result of those studies was to specify that the only absolute temperature is not enough for classifying the foot state. Therefore, some works [2] investigated the full temporal thermal signal after a cold stimulus. The rewarming of the foot was recorded with an IR camera. Then, a homemade bio-heat model, including a micro perfusion parameter, was used to fit the signal during some seconds. They conclude, comparing controls and DM population, that a difference between these two populations seems to appear regarding the micro perfusion parameter on the metatarsal head, while all the other parameters remain still.

Based on these works, and a previous study made on skin thermal behaviour [3], a methodology using IR thermal imaging, during a transient rewarming after a cold stimulus beneath the foot, is proposed in this paper. A first study was conducted at the hospital with a sample of patient with DF. A particular attention was paid to the post-processing (including calibration, correlation and filtering) of the recorded IR images. An inverse method was then used for the parameters identification of a one-dimensional bio-heat model. The thermophysical parameters and blood perfusion parameter of this model are finally compared to the clinical classification of the patients.

\section{Patients and methods}

The study includes 10 patients with diabetes (type I or II) but no peripheral neuropathy or arteriopathy, 5 diabetic patients with neuropathy and/or arteriopathy and/or ulceration history, 5 healthy persons (control), for a total of 20 tested peoples. Exclusion criteria were as follows: other cause of peripheral neuropathy or arteriopathy, lower limb amputation, current foot ulceration and other systemic disease that might interfere with body temperature.

All subjects were studied in an air-conditioned room maintained at a temperature between 21 and $23^{\circ} \mathrm{C}$. Patients were asked to remove footwear and socks and to wait 5 minutes for the adaptation of their bare skin to indoor conditions. Subsequently, a cold stimulus was applied beneath the foot. Two kind of cold stimulus were tested, the first one was local and the second global. The local one consisted of a cold $\left(10^{\circ} \mathrm{C}\right)$ stainless steel cylinder applied under the first metatarsal head during 30 seconds. The global stimulus was an ambient plate applied beneath the foot during 30 seconds. The thermal response was recorded during 5 minutes just after the removal of the stimulus. The first stimulus enabled the local parameter extraction under a specific part of the feet and the thermal body reaction observation around this area. The second stimulus enabled the feet mapping of those parameters and the visualization of the support area of each patient. A Cedip MW infrared camera (Titanium 640x512 pixels) was used for the thermal observations. 


\section{Bio-heat model}

The bio-heat model used in the present work is based on the heat equation with a specific heat source for the blood micro-perfusion system, i.e.the Pennes's heat source [4] as shown in Eq. (1). Skin and soft tissue conduction are commonly low $\left(\mathrm{k}<0.5 \mathrm{~W} \cdot \mathrm{m}^{\wedge}(-1) \cdot \mathrm{K}^{\wedge}(-1)\right)$. Therefore a one-dimensional model in the depth direction was chosen to analyse the thermal response at the skin surface around the centre of the stimulus application area. Moreover, the model was supposed to be far enough from a vein or artery. The spatial domain in the thickness direction was limited to $10 \mathrm{~mm}$ deep, averaging the several layers under the skin surface (epidermis, dermis, fat and muscle) to form an equivalent material noted tissue hereafter. Considering homogeneous thermophysical properties of this tissue material in this domain, the temperature $T(x, t)$ of the tissue in a spatial point located in the thickness position $x$ at time $t$ can be modelled by the following $1 \mathrm{D}$ bio-heat equation.

$$
\rho . C \frac{\partial T}{\partial t}-k \nabla^{2} T=\rho_{b} C_{b} \omega .\left(T_{a}-T\right)
$$

where $\mathrm{k}$ is the tissue thermal conductivity, $\rho$ the density, $\mathrm{C}$ the tissue heat capacity, $\omega$ the blood perfusion rate (in $\left.\mathrm{s}^{\wedge}(-1)\right)$ parameter and Ta the inner body temperature.

\section{Preliminary results}

The identification of the different parameters of the model (inner temperature $\left(T_{a}\right)$, tissue homogenous thermal conductivity $(k)$ and blood perfusion rate $(\omega))$ was done with a steepest-descent algorithm, based on experimental data. The obtained values were compatible with physiological values found in the literature for each terms. An example of such identification is plotted in figure 1 .

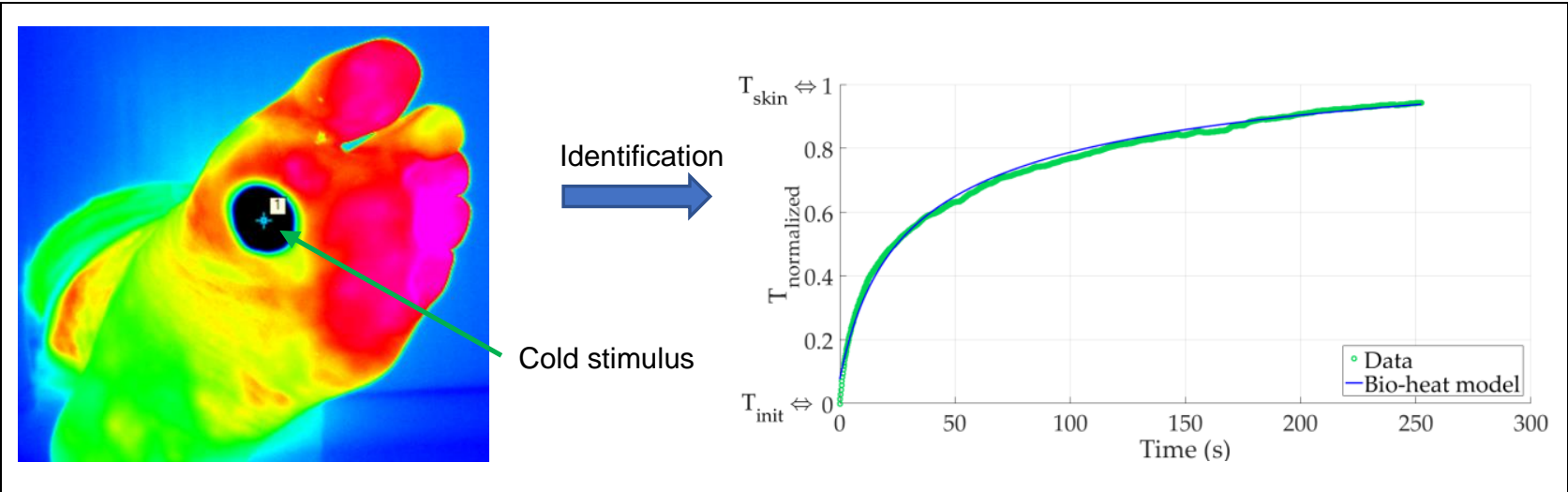

Fig. 1: (left) Thermal image recorded just after the local cold stimulus. (right) Experimental (dot) and model (continuous) results of the rewarming response in a normalized scale: $T_{\text {normalized }}=\left(T-T_{\text {init }}\right) /\left(T_{\text {skin }}-T_{\text {init }}\right)$

After the identification of the first experimentation in the hospital, the different parameters have been crossed with the clinical classification of each patient. It seems that the only micro-perfusion term was not enough for an accurate classification of the foot state, so the influence of the others parameters have to be investigated. Moreover, future work in the hospital on new subject will be conducted in order to evaluate the specificity and sensitivity of this dynamic method to analyse the risks of Diabetic Foot.

\section{REFERENCES}

[1] P. Sousa et V. Felizardo, «A review of thermal methods and technologies for diabetic foot assessment,» Expert Review of Medical Devices, vol. 12, pp. 439-448, 2015.

[2] G. Iven et V. Chekh, «Non-contact Sensation Screening of Diabetic Foot Using Low Cost Infrared Sensors,» International Symposium on Computer-Based Medical Systems, pp. 479-480, 2014.

[3] R. Domoina et J. Franck, «Etude expérimentale et numérique de la diffusion de la chaleur, Congrès Français de Mécanique, 2009.

[4] H. H. Pennes, «Analysis of tissue and arterial blood temperatures in the resting human forearm.,» Journal of applied physiology, vol. 1, pp. 93-122, 1948. 